\title{
Reference-free Automated Magnetic Sensor Calibration for Angle Estimation in Smart Knee Prostheses
}

\author{
Arash Arami, Member, IEEE, Julien David Rechenmann, and Kamiar Aminian, Member, IEEE
}

\begin{abstract}
In this work, we present a method for automated calibration of an implanted anisotropic magnetoresistive (AMR) sensor for measuring the internal-external rotation (IE) in a prosthetic knee without using any reference measurement. The measurement system consists of a permanent magnet and a 2-D AMR sensor configured and embedded into the prosthesis. Using the physical relation between the measurements of channels of sensor, we proposed a reference-free calibration method through the optimization. The parameters of the measurement model of the sensor obtained via the calibration were directly used in an angle estimator. Two different optimization approaches were tested, and the resultant estimates were compared to the reference angle with the expected \pm standard deviation of error of $-1.69^{\circ} \pm 0.65^{\circ}$ and $-1.17^{\circ} \pm 1.13^{\circ}$. The reference-free calibrated estimators were also compared to the reference-dependent leastsquare estimators. This latter comparison showed an offset error difference among the estimators while the coefficients of determination for the both reference-free and referencedependent estimators were high, i.e. larger than 0.91 . The good performances were maintained even when using a small portion of data for the optimization. The sensor-magnet misalignment effect was also investigated. The misalignment slightly worsened the mean error of the estimates, but neither dramatically affected the variance of error nor the coefficient of determination.
\end{abstract}

Index Terms-Knee rotations, Smart knee prosthesis, Anisotropic Magnetoresistive sensor, Optimization

\section{INTRODUCTION}

$\mathrm{K}$ NEE arthroplasty consists of partially or totally replacing the knee joint by a prosthesis. This operation is the most successful and widely performed solution for end-stage knee osteoarthritis or rheumatoid arthritis. Each year more than a million knee joints are replaced [1] mainly to relieve the pain and restore the function, however the prostheses do not provide any feedback information about their functional states. Any defect due to inaccuracy in the surgery or further complications is left to be detected by the subjective

\footnotetext{
${ }^{\mathrm{T}}$ his paragraph of the first footnote will contain the date on which you submitted your paper for review. This work was funded by Nano-Tera (SNF20NAN1_123630).

A. Arami and K. Aminian are with laboratory of movement analysis and measurement at Ecole Polytechnique Fédéral de Lausanne (EPFL), Lausanne, Switzerland (emails: arash.arami@epfl.ch and kamiar.aminian@epfl.ch).

J. D. Rechenmann is with school of life science at Ecole Polytechnique Fédéral de Lausanne (EPFL), Lausanne, Switzerland.
}

interpretation of patients' pain level or movement difficulties.

To provide an objective evaluation of the prosthetic knee function, the measurements of the forces acting on it and its 3D kinematics are needed. The knee contact forces need to be measured in-vivo due to the lack of accurate models and impossibility of estimating the exact muscle forces acting on the knee [2]-[4]. In case of kinematic measurements, different techniques were used to measure the knee rotations namely Flexion-Extension, Abduction-Adduction and InternalExternal (IE) rotations. Stereophotogrammetry techniques with skin-fixed marker and motion capture system are widely used but are restricted to laboratory space, and also suffers from soft tissue artifact (STA) [5], [6]. The movements of skin due to the muscle contractions, soft tissue inertia and vibration add a subject- and activity-dependent error (STA) to the overskin measurement systems. The range of error is very high particularly for knee internal-external rotations with an RMS reaching $4^{\circ}$ [7]. The importance of monitoring the IE rotation has been shown in case of mobile-bearing prosthesis [8], [9] which are designed to reduce the polyethylene wear via a better stress distribution. Body-fixed inertial sensors (e.g. a combination of gyroscopes and accelerometers) were used for in-field gait analysis [10]-[12], kinematics measurements [13]-[16], and tested on a number of subjects. In addition to STA, the kinematics measurement techniques suffer from the integration drift of angular velocity, inaccuracies due to estimation of the rotation axis and its relative position to the sensors and their limitations to measure IE angles [15].

Model-based radiostereometry based on biplane fluoroscopy was proposed to provide kinematic measurements of prosthetic knee without STA with an approximate precision of $0.6^{\circ}$ for IE rotation [17]. Similar precision was reported for the F.I.R.S.T prosthesis (Symbios, $\mathrm{CH}$ ) which was used in our study [18]. Nevertheless, the fluoroscopic methods remain as laboratory-based that suffer from constrained movement range and limited number and duration of measurements due to their small capture volume, and $\mathrm{X}$-ray radiation respectively.

Recently some prototypes for the new generation of the prostheses have been designed including the instrumented knee prostheses [19]-[23]. The integrated sensors in those prostheses can measure the knee contact forces and knee rotations and provide very useful information about the prosthetic joint function, and revealing the possible defects such as unbalanced forces and abnormal kinematics [21]. 
Integrating the kinematic sensors into the prosthesis can provide STA-free kinematic estimations in daily conditions. In one of the recent studies, a configuration of anisotropic magnetoresistive (AMR) sensors and a permanent magnet, integrated into the knee prosthesis, was used to estimate the knee internal-external (IE) rotation [24]. The AMR sensors were chosen due to their high resolution [25], and their success in measuring the $3 \mathrm{D}$ orientation and position of a magnet [26] in different biomedical applications such as gastrointestinal motility studies [27], and step detection during walking [28]. The main reason to focus on IE rotation is its clinical importance as well as the difficulty of its external measurement due to the STA. The angle estimators in that work, is needed to be calibrated (trained) with the reference data which come from pre-operation measurements in a knee simulator. However, the post-operative performance of the estimators is subject to many inaccuracies due to the possibility of inexact placement of prosthetic components during the surgery, inaccuracy in fabrication and placement of the sensors and magnet in the smart prosthesis or the sensor performance changes with time. A post-operative recalibration of the angle estimators is thus required. Any skin-mounted sensors cannot be used as the reference system for training the estimators since they are STA-affected, and it deteriorates all the benefits of the implantable kinematic sensors. X-ray fluoroscopic measurement would be a possible solution for recalibration; however it could only train the estimators in a very restricted set of movements and in laboratory conditions. The X-ray data processing is very cumbersome [29], and would be challenging since the electronics in the PE would be captured in the images and worsen the accuracy of the kinematic estimations.

In this work, we proposed an automated reference-free calibration method for the integrated AMR sensor in smart knee implants. The method is based on an assumed physical model for the sensor, and a constraint optimization only on the implanted AMR sensor's measurements. This reference-free calibration can be applied mainly for the automatic training of IE angle estimators or recalibrate them after implantation.

\section{Method AND Materials}

The typical parts to be implanted during a total knee arthroplasty are tibial (TP) and femoral (FP) parts and an ultra-high-molecular-weight polyethylene insert (PE). We used the F.I.R.S.T prosthesis (Symbios, $\mathrm{CH}$ ), that is posteriorstabilized mobile-bearing knee prosthesis (Fig. 1). The used magnetic sensor was a 2D AMR sensor (HMC1512, Honeywell, USA) which is sensitive to the direction of the magnetic flux passing through it. The sensor measures the direction of magnetic field in two bridges, which are physically rotated according to each other, outputted in two channels denoted by $S 1_{1}$ and $S 1_{2}$. A devised measurement system was considered to measure IE rotations of the prosthetic knee. The minimally-designed system consisted of a permanent magnet placed in TP and an AMR sensor located on the axis of rotation of PE (Fig. 2) [24]. As long as the magnet and sensor are in the same plane pointing to each other and their relative angle is small, the measurement equations can be considered as sinusoidal equations (1) and (2) [24], where $a_{1}, a_{2}$ and $k_{1}, k_{2}$ are gains and offset of the models.

$$
\begin{aligned}
& S 1_{1}=a_{1} \sin (\theta)+k_{1} . \\
& S 1_{2}=a_{2} \cos (\theta)+k_{2} .
\end{aligned}
$$

\section{A. Formulation of the reference-free calibration problem}

We first assumed that (1-2) are preserved. Then using the relation between the channel measurements (3), we defined an optimization problem independent of IE angles $(\theta)$.

$$
\sin ^{2}(\theta)+\cos ^{2}(\theta)=\left(\left(S 1_{1}-k_{1}\right) / a_{1}\right)^{2}+\left(\left(S 1_{2}-k_{2}\right) / a_{2}\right)^{2}=1 .
$$

By expanding (3), a parametric function (4) was derived over a set of parameters $(\underline{\beta})$.

$$
f(\underline{\beta})=\beta_{1} S 1_{1}^{2}+\beta_{2} S 1_{2}{ }^{2}+\beta_{3} S 1_{1}+\beta_{4} S 1_{2}+\beta_{3}{ }^{2} / 4 \beta_{1}+\beta_{4}{ }^{2} / 4 \beta_{2}-1 .
$$

where $\beta_{1}=1 / a_{1}^{2}, \beta_{2}=1 / a_{2}^{2}, \beta_{3}=-2 k_{1} / a_{1}^{2}, \beta_{4}=-2 k_{2} / a_{2}^{2}$. Alternatively, (4) can be rewritten as (5). The parameters can be obtained through solving the optimization problem (6).

$$
\begin{aligned}
& f(\underline{\beta})=\beta_{1} S 1_{1}{ }^{2}+\beta_{2} S 1_{2}{ }^{2}+\beta_{3} S 1_{1}+\beta_{4} S 1_{2}+\beta_{5}-1 . \\
& \underline{\beta}^{*}=\arg \min _{\underline{\beta}}\|f(\underline{\beta})\| \\
& \text { s.t. } \quad \beta_{1}, \beta_{2}>0 \text { and } \beta_{5}=\beta_{3}{ }^{2} / 4 \beta_{1}+\beta_{4}{ }^{2} / 4 \beta_{2} .
\end{aligned}
$$

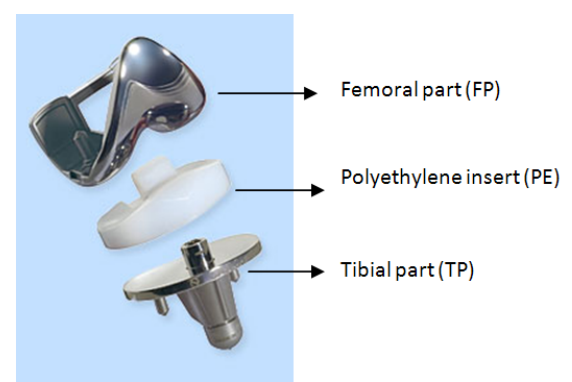

Fig. 1. F.I.R.S.T. knee prosthesis (Symbios Orthopédie SA, CH).
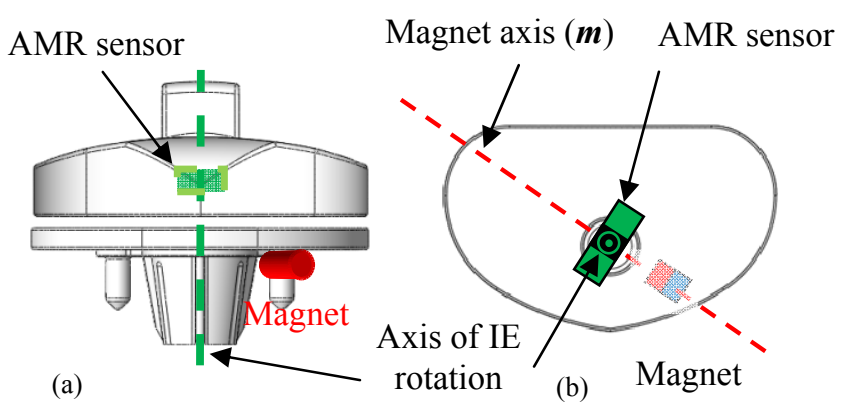

Fig. 2. IE measurement system. (a) a front view of the TP and PE, where the magnet and AMR sensor placement are shown as well as the axis of IE rotation. (b) top view showing the magnet axis and the axis of IE rotation. 
This constraint optimization can be reformulated as a Lagrange multiplier and be solved by optimization tools. This way, without using any reference angle information, the obtained solutions for $\underline{\beta}$ parameters $\left(\underline{\beta}_{\text {ref free }}\right)$ can be converted into $\alpha_{1, \text { reffree }}$ and $k_{1, \text { ref free }}$ to build an angle estimator.

$$
\hat{\theta}_{\text {ref-free }}=\sin ^{-1}\left(\left(S 1_{1}-k_{1, \text { ref-free }}\right) / a_{1, \text { ref-free }}\right) .
$$

\section{B. Optimization approaches}

To solve the optimization problem (6) two different approaches were tested. A quasi-Newton algorithm applied in a Lagrange multiplier setup of (6) as an efficient method to find local minima (stationary point) of a function; however it cannot escape a local minimum. Also a global optimization algorithm based on multiple ant colonies (MHACIE) [30] to heuristically search the space and find local minimums of a cost function defined upon (6). The global optimization algorithms such as ant colony work based on some guided random search on the parameter space which may result in longer convergence time. These algorithms do not rely on finding a stationary point but to minimize a cost function. They thus deal nicely with local minima problems.

\section{1) Quasi-Newton algorithm (QN)}

This method finds the local minima/maxima of a function, considered as the stationary points where the gradient of the function is zero. Quasi-Newton (QN) method is based on Newton method which, particularly in higher dimensions, uses the gradient and the Hessian matrix of the function to be minimized. In QN method, the Hessian matrix is estimated based on successive gradient vectors [31]. The function was then estimated by a second order Taylor series (8).

$$
f\left(x_{n}+\Delta x\right) \approx f\left(x_{n}\right)+\nabla f\left(x_{n}\right)^{T} \Delta x+1 / 2 \Delta x^{T} h\left(x_{n}\right) \Delta x .
$$

where $\nabla f$ and $h$ are gradient of function $f$ and its hessian approximation respectively. To estimate the Hessian, Broyden-Fletcher-Goldfrab-Shanno method (BFGS) was used [31]. The initial values of $\underline{\beta}$ was first randomly selected, but then fixed to an appropriate one to obtain a better local minimum of the norm of the function $f(\underline{\beta})(6)$.

\section{2) Global optimization using MHACIE}

The global optimization algorithms are the other class of algorithms which can solve also the non-convex optimization problems. To apply them for finding the optimal parameters of the problem (6), we first defined a cost function $(\operatorname{Cost}(\beta))$ to fuse the constraints into the main objective (9). Here the constraint was plugged two times, once multiplied by the main objective to boost the effect of unsatisfied constraint in an exponential way, and once as a summation to be influential

$$
\begin{array}{r}
\operatorname{Cost}(\underline{\beta})=\|f(\underline{\beta})\|^{2} \times \exp \left(\left(\beta_{3}^{2} / 4 \beta_{1}+\beta_{4}^{2} / 4 \beta_{2}-\beta_{5}\right)^{2}\right) \\
+\left(\beta_{3}^{2} / 4 \beta_{1}+\beta_{4}^{2} / 4 \beta_{2}-\beta_{5}\right)^{2}
\end{array}
$$

when $\|f(\beta)\|$ is small. The resultant cost is a continuous and smooth function, which facilitates the optimization process.

We solved this problem via Ant Colony Optimizations (ACO) [32], i.e. meta-heuristic methods inspired by foraging behavior of ants and their communication via pheromone deposits. The problem is first coded into a graph consists of the nodes connected with the edges where the ants probabilistically pass and provide a candidate result. In our setup each parameter $\left(\beta_{i}\right)$ was binary coded by 12 nodes (bits) in a fully connected graph. Adding two terminal nodes (start and finish), the problem graph was obtained. Each ant chooses a sequence of edges of the graph to pass based on a probabilistic rule built upon the pheromone quantity in the neighbor edges $\left(\tau_{i j}\right)$ and a heuristic for goodness of edges $\left(\eta_{i j}\right)$. The probability of passing the $e d g e_{i j}$ (from node $_{i}$ to $\operatorname{node}_{j}$ ) is:

$P_{i j}=\tau_{i j}\left[\eta_{i j}\right]^{\gamma} / \sum_{\forall u \in \text { neighbor }(i)} \tau_{i u}\left[\eta_{i u}\right]^{\gamma}$

where $\gamma$ is an adjustable importance factor, neighbor(i) is a set of all nodes which are connected to $i^{\text {th }}$ node. In our setup, no heuristic was used, and all $\eta_{i j}$ values were thus set to 1 . The initial pheromones were randomly generated prior to the optimization. All the nodes' values were initially set to 0 and as soon as an ant reaches a node its value set to 1 . This way, each time an ant starts from one terminal node and finishes at the other, a candidate solution is obtained via decoding the values of the nodes correspond to each parameter $\beta_{i}$. Based on the goodness of the result, i.e. the Cost obtained from $\beta_{i}$ s, different strategies can be used for the pheromone deposition and update over the problem graph. This deposition of pheromone is in competition with the vaporization of all pheromones and results in a convergence to the more frequently deposited paths, i.e. the optimal or near optimal solution. For instance in ant system optimization [32], a local update applied after each ant passes in the graph to reduce the pheromone of the traveled edges ( $\varepsilon$ is a time step, and $t+\varepsilon$ represents just after an ant passes an edge at time $t$ ):

$\tau_{i j}(t+\varepsilon)=(1-\rho) \tau_{i j}(t)+\rho \tau_{0}$

where $\rho$ and $\tau_{0}$ are the local pheromone vaporization rate and the pheromone level constant. When all the ants of a colony passed the graph, a global pheromone update applied to increase the best path pheromone and vaporize a fraction of the rest of pheromones (12), where $\mu$ is the vaporization rate and $\Delta \tau$ is proportional to the pheromone deposition.

$$
\begin{aligned}
& \tau_{i j}(t+1)=(1-\mu) \tau_{i j}(t)+\mu \Delta \tau_{i j} \\
& \Delta \tau_{i j}= \begin{cases}1 / \operatorname{Cost}(\underline{\beta}) ; & \text { if edge } e_{i j} \in \text { best path } \\
0 & \text { otherwise }\end{cases}
\end{aligned}
$$

There are multiple parameters to be tuned in each ACO algorithm which are task-dependent and have significant effects on the efficiency and performance of the optimization algorithm [33]. In this study we used a variant of ACO called 
Multiple Heterogeneous Ant Colonies with Information Exchange (MHACIE) [30]. MHACIE is an optimizer build upon multiple ant colonies with different pheromone deposition policies (different parameters) to relax the need for tuning the ACO parameters for each arbitrary problem. In MHACIE, each colony attempts to solve the problem in the individual attempt steps, and then colonies randomly communicate their best found results in the communication steps. The communication steps will result in the convergence of all colonies after a sufficient number of iterations. In this study four different colonies were used in the MHACIE similar to the original version [30], and in each run we let the algorithm to perform 100 iterations to find the parameters $\underline{\beta}$.

\section{Validation}

To validate the reference-free calibration method and evaluate the performance of the resultant IE angle estimators, we compared the result to the reference kinematics measured by a stereophotogrammetry motion capture system, and to the reference-dependent trained IE estimators proposed earlier [24]. The instrumented knee prosthesis was fixed in a manually-operated knee simulator able to perform different rotations. The motion capture system, synchronized with the AMR sensor, was used to reconstruct the exact motion of the prosthesis (the reference rotations). The motion capture system consisted of four Mx3+ cameras (Vicon, UK) and four reflective markers attached to the simulator fixed body, and a rotating disk fixed to the TP (Fig. 3). Using the position of these markers, the reference angle was estimated based on the angle between vectors $l_{1}$ and $l_{2}$ [24]. The rotations were performed in a range of $\left[\begin{array}{lll}-11.31^{\circ} & 9.71^{\circ}\end{array}\right]$. The obtained expected resolution of the reference IE angle was $0.13^{\circ}$ while the worst case resolution in the range of performed rotation was $0.16^{\circ}$. In addition, different reference-dependent estimators ( $\hat{\theta}_{1}$ and $\hat{\theta}_{2}$ ) were designed and trained with the reference data (13-14).

$\hat{\theta}_{1}=\sin ^{-1}\left(\left(S 1_{1}-k_{1}\right) / a_{1}\right)$

$\hat{\theta}_{2}=w_{0}+w_{1} S 1_{1}+w_{2} S 1_{2}$

We used a cross-validation setup in which different continuous subsets of data were randomly selected for finding the best parameters of the estimators, and then the estimators were validated over the rest of data. By repeating the random subset-selection cross validation 100 times for six different train subset sizes over the IE rotation data, we evaluated the performance of the angle estimators. This evaluation included the effect of calibration over different percentages of measurements, or better said over poorer or richer datasets. The performances were reported using expected value and standard deviation of the mean error, the standard deviation of error, RMS error and coefficient of determination $\left(R^{2}\right)$.

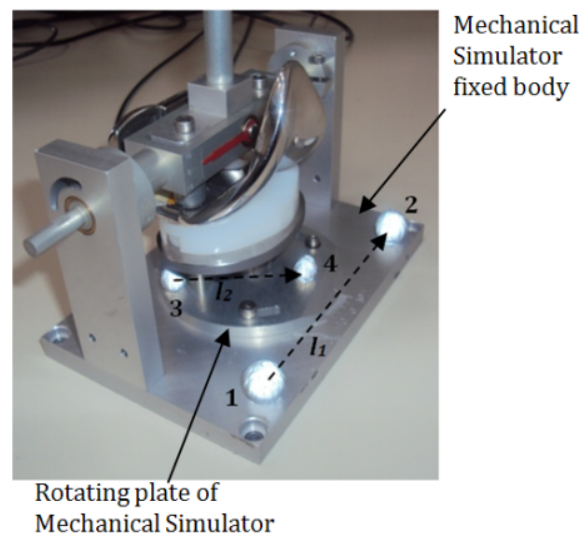

Fig. 3. Marker placement in the knee simulator [24]: after reconstruction of reflective markers the vectors $l_{1}$ and $l_{2}$ were used to estimate the reference IE.

\section{Magnet-sensor misalignment analysis}

The misplacement of the magnet or the imperfections in the fabricated sensor such as any inaccuracies in the relative orientation of the sensor's sensitive bridges (plates) can worsen the quality of the reference-free estimates. The effect of magnet-sensor misalignment is one of the most crucial errors since it affects the underlying assumption of the reference-free calibration showed in (1-3). Assuming the magnet-sensor misalignment represented by the angle $\varphi$, as shown in Fig. 4, the magnetic field in the sensor location was calculated via magnetic dipole equation (15), where $\mu_{0}$ is the magnetic permeability of air (or vacuum), $m_{i}$ is the magnetic moment of the magnet, and $r_{i}$ is the relative position of the sensor and magnet. Plugging the misalignment angle $\varphi$ and the corresponding rotation matrix $R(\varphi)(16)$, the magnetic field in the sensor's location was derived (17).

$B=\frac{\mu_{0}}{4 \pi}\left(\frac{3\left(m_{i} \cdot r_{i}\right) r_{i}-\left(r_{i} \cdot r_{i}\right) m_{i}}{\left|r_{i}\right|^{5}}\right)$.

$R(\varphi)=\left(\begin{array}{cc}\cos \varphi & -\sin \varphi \\ \sin \varphi & \cos \varphi\end{array}\right)$

$B=\frac{\mu_{0}}{4 \pi}\left(\frac{3\left(\left|m_{i}\right|\left|r_{i}\right| \cos \varphi\right) r_{i}-\left(\left|r_{i}\right|\left|m_{i}\right|\right) R(\varphi) r_{i}}{\left|r_{i}\right|^{5}}\right)$.

Considering the frame of the sensor (Fig. 4), $r_{i}$ was decomposed to its projection on the axes.

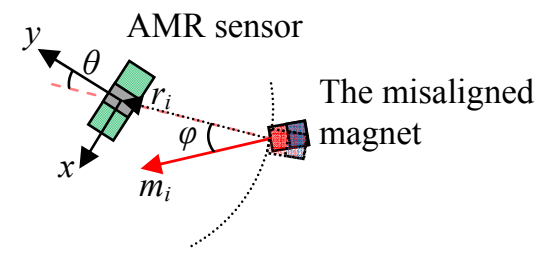

Fig. 4. Misaligned magnet and sensor (when the magnet rotates around the sensor on a half circle during the IE rotation). 
$r_{i}=\left|r_{i}\right|(\sin \theta \quad \cos \theta)^{T}$.

The resultant magnetic field $(B)$ is shown in (19), which suggested an alternation of the measured magnetic field through the AMR sensor due to the misalignment $\varphi$.

$$
B=\frac{\mu_{0}\left|m_{i}\right|}{2 \pi\left|r_{i}\right|^{3}}\left(\sin \theta \cos \varphi+\frac{\cos \theta \sin \varphi}{2} \cos \theta \cos \varphi-\frac{\sin \theta \sin \varphi}{2}\right)^{T} \text {. }
$$

In this case, the measured signal (e.g. $S 1_{l, \varphi}$ ) theoretically corresponds to (20).

$$
S 1_{1, \varphi}=a_{1}{ }^{\prime} B_{x}+k_{1}{ }^{\prime}=\alpha a_{1}{ }^{\prime}\left(\sin \theta \cos \varphi+\frac{\cos \theta \sin \varphi}{2}\right)+k_{1}{ }^{\prime} .
$$

Comparing (20) to (1-2), we reformulated the measurement in the misaligned condition (21-22).

$$
\begin{aligned}
& S 1_{1, \varphi}=S 1_{1} \cos \varphi+S 1_{2} \sin \varphi / 2 . \\
& S 1_{2, \varphi}=S 1_{2} \cos \varphi-S 1_{1} \sin \varphi / 2 .
\end{aligned}
$$

When $\varphi=0$, these equations reduced to (1-2) -the gains $a_{I}$ and $a_{2}$ in those equations cancel the effect of the coefficient of $\left(\mu_{0}\left|m_{i}\right| /\left(2 \pi\left|r_{i}\right|^{3}\right)\right)$ which was appeared in (19)- and (3) was maintained. Applying the optimization when $\varphi \neq 0$, resulted in new parameters (offset and gains) that satisfy (3) and were different from the previously obtained parameters. The new parameters could improve or worsen the result of the IE angle estimation. The angle estimator (7) was reformulated for misaligned magnet-sensor (23), where $a_{1, \text { reffree }}$ and $k_{1, \text { reffree }}$ represent the new coefficients obtained from optimization.

$$
\hat{\theta}_{\text {ref-free }}=\sin ^{-1}\left(\left(S 1_{1, \varphi}-k_{1, \text { ref-free }}^{\prime}\right) / a_{1, \text { ref-free }}^{\prime}\right) .
$$

The error induced by this misalignment was estimated by simulating the measurements of sensors in a small range of $\varphi$. The optimization was then performed to get new referencefree estimators, changing $\varphi$ every degree from $-5^{\circ}$ to $5^{\circ}$. For each misalignment angle the optimization was performed on $70 \%$ of randomly selected data 100 times and the mean error, standard deviation of error, RMS error and $\mathrm{R}^{2}$ were reported.

\section{RESULTS}

Applying the QN algorithm over constraint optimization problem shown in (6), different results were obtained starting from different initial $\underline{\underline{\beta}}$. This corroborates to the non-convexity of the optimization problem. The solutions are thus suboptimal. We did a search over different initial $\underline{\beta}_{0}$ to obtain better results. For instance, the results for three different $\underline{\beta}_{0}$ when using all data for optimization are shown in Table I. Fixing $\underline{\beta}_{0}=[200,20,0,0]$, the performance of the referencefree calibration using the QN method against the reference angle was validation (Fig. 5.a). Although by starting from the fixed $\underline{\beta}_{0}$ no remarkable variation of performance indices was observed, a noticeable variation of the performance quality of the reference-free calibration was observed when it started from different initial points. The performance did not change when utilizing different percentage of data in solving the optimization to find the parameters, i.e. the calibration.

Using the MHACIE, the algorithm was not sensitive anymore to the $\underline{\beta}_{0}$, but it took more time to find the nearoptimal $\underline{\beta}$. The results are shown in Fig 5.b. The results of both optimization methods were very close. The performances of both methods were not influenced when performing the optimization on larger portions of data. There was an expected error of $-1.7^{\circ}$ for $\mathrm{QN}$ optimization from the fixed $\underline{\beta}_{0}$, while this error is smaller in case of MHACIE optimization, i.e. less than $-1.3^{\circ}$ with larger variations, as a result of random initial points. The expected standard deviations of error were below $0.7^{\circ}$ and $1.2^{\circ}$ for the case of $\mathrm{QN}$ and MHACIE optimizations respectively. The coefficient of determination for both cases were relatively high, i.e. approximately 0.93 and 0.91 for QN and MHACIE respectively.

The reference-dependent estimates, i.e. (13-14), were also tested in the same cross validation setup Fig. 6, and compared with the reference-free estimators. The validation results of all mentioned estimation methods for the case of $30 \%$ test data are summarized in Table II. Both reference-dependent estimators outperformed the reference-free ones. The variation of performance in the cases of reference-dependent estimators is noticeable when training on different percentages of data.

The effects of different magnet-sensor misalignments in range of $-5^{\circ}$ to $5^{\circ}$ were simulated, and the results were depicted in Fig. 7. Based on these results the misalignment worsened the mean error up to $90 \%$, while the standard deviation of error was not noticeably changed. The IE angle estimation offset and RMS was worsened for extreme negative misalignments, but improved for positive misalignments. It is noteworthy that in the observed worst case, i.e. $-5^{\circ}$ misalignment, only $0.9^{\circ}$ was added to the RMS error of IE angle comparing to the well-aligned magnet and sensor case. The $\mathrm{R}^{2}$ was not worsened for positive misalignments (expected $R^{2}>0.9$ ), while negative misalignments slightly worsened it, e.g. in the case of $-5^{\circ}$ and $-4^{\circ}$ misalignments $\mathrm{R}^{2}$ were $0.79 \pm 0.06$ and $0.82 \pm 0.09$ respectively.

\section{DISCUSSION}

\begin{tabular}{|c|c|c|c|}
\hline$\underline{\beta}_{0}$ & Mean(error) ${ }^{\circ}$ & $\mathrm{SD}$ (error) ${ }^{\circ}$ & $\mathrm{RMS}$ (error) ${ }^{\circ}$ \\
\hline$[50,1,30,30,20]$ & 0.27 & 3.28 & 3.29 \\
\hline$[150,35,10,10,0]$ & -1.25 & 0.99 & 1.56 \\
\hline$[200,20,0,0,0]$ & -1.70 & 0.65 & 1.82 \\
\hline
\end{tabular}

In this work, we presented a reference-free calibration method to estimate the internal-external rotations using an

TABLE I. EFFECT OF INITIAL POINT ON PERFORMANCE OF THE OBTAINED REFERENCE-FREE ESTIMATORS BY QUASI-NEWTON METHOD

Different initial parameters for the optimization led to different results for reference-free calibration based on QN method. 

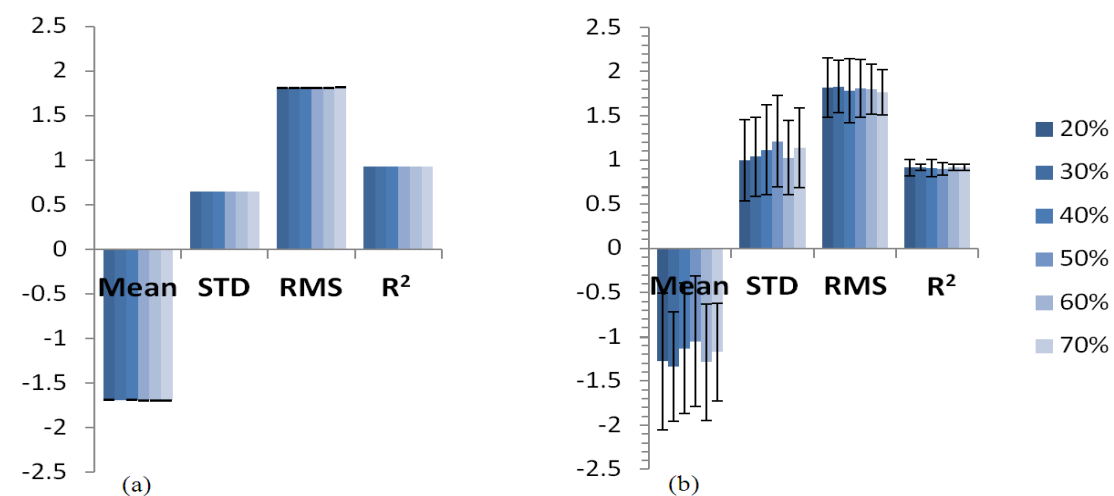

Fig. 5. Cross validation results (results over test data) of reference-free algorithms, when using different percentage of data (from $20 \%$ to $70 \%$ ) as the training set. Reference-free IE estimation (a) using QN (starting from the mentioned appropriate initial value), (b) using MHACIE.
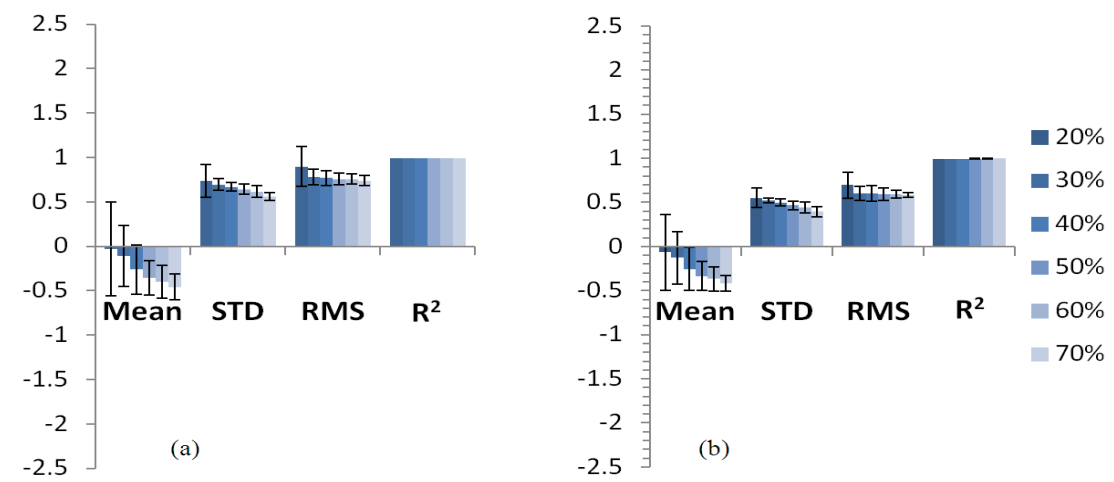

Fig. 6. Cross validation results (results over test data) of reference-dependent algorithms, when using different percentage of data (from $20 \%$ to $70 \%$ ) as the training set: (a) $\hat{\theta}_{1}$, (b) $\hat{\theta}_{2}$.

TABLE II. COMPARISON OF REFERENCE-FREE AND REFERENCE-DEPENDENT ESTIMATORS

\begin{tabular}{lcccc}
\hline \hline \multicolumn{1}{c}{ Estimators } & Mean(error) & RD(error $)^{\circ}$ & $\mathrm{RMS}^{\circ}$ (error) & $\mathrm{R}^{2}$ \\
\hline$\hat{\theta}_{\text {ref_free }}(\mathrm{QN})$ & $-1.69 \pm 0.01$ & $0.65 \pm 0.00$ & $1.81 \pm 0.00$ & $0.93 \pm 0.00$ \\
$\hat{\theta}_{\text {ref_free }}(\mathrm{MHACIE})$ & $-1.17 \pm 0.55$ & $1.13 \pm 0.45$ & $1.77 \pm 0.28$ & $0.91 \pm 0.04$ \\
\hline$\hat{\theta}_{1}$ & $-0.46 \pm 0.14$ & $0.56 \pm 0.05$ & $0.74 \pm 0.06$ & $0.99 \pm 0.00$ \\
$\hat{\theta}_{2}$ & $-0.42 \pm 0.09$ & $0.39 \pm 0.06$ & $0.58 \pm 0.03$ & $0.99 \pm 0.00$ \\
\hline \hline
\end{tabular}

The $\overline{\text { resultant reference-free and reference-dependent estimators were compared when their parameters obtained using } 70 \% \text { of randomly selected data and }}$ tested on $30 \%$ data (100 times sub-sampling cross validation).

embedded magnetic sensor in instrumented knee prostheses. The method relies on the physical relation between the measurements of channels of an AMR sensor placed in the polyethylene insert while a magnet in tibial part always pointing at it. A constraint optimization problem was formulated, which does not explicitly depend on the IE angle. We solved the optimization in two different approaches, and obtained good results in angle estimation even when using a small percentage of data. The so-called Quasi-Newton (QN) optimization, finds a local minima of the parametric function $f(\underline{\beta})$. Since the function $f$ is non-convex, the $\underline{\beta}_{0}$ affects the converged solution and leads to a variation of RMS errors from $1.56^{\circ}$ to $3.29^{\circ}$ for three different $\underline{\beta}_{0}$. This suggests the need for repeating the optimization from different initial points or using a prior knowledge. The initial parameter approximation could be achieved through the calibration of the sensor before implantation in a knee simulator. Although the condition in the knee simulator is not totally realistic, it is potentially a proper initial point. Starting from that initial point, this optimization resulted in precise IE estimates with expected mean and standard deviation of error of $-1.69^{\circ}, 0.65^{\circ}$ and $\mathrm{R}^{2}$ of 0.93 . This estimator was robust to the amount of data used for the optimization.

Multiple heterogeneous ant colonies with information exchange (MHACIE) is a meta-heuristic global optimization which converges to the global minimum or a near-optimal local minimum of the function in a limited number of iterations. This method resulted in similar angle estimators to the previous method with expected mean and standard deviation of error of $-1.17^{\circ}$ and $1.13^{\circ}$, and an $\mathrm{R}^{2}$ of 0.91 . However these results were obtained when starting the optimization each time from a random initial point, confirming that this method -in contrast to the QN optimization- is independent of the initial value of the parameters. A clear benefit of this method is that there is no need for prior knowledge about the approximate parameters of the estimators and consequently no need for a pre-calibration. Similarly to the QN optimization, the resultant estimators by MHACIE 

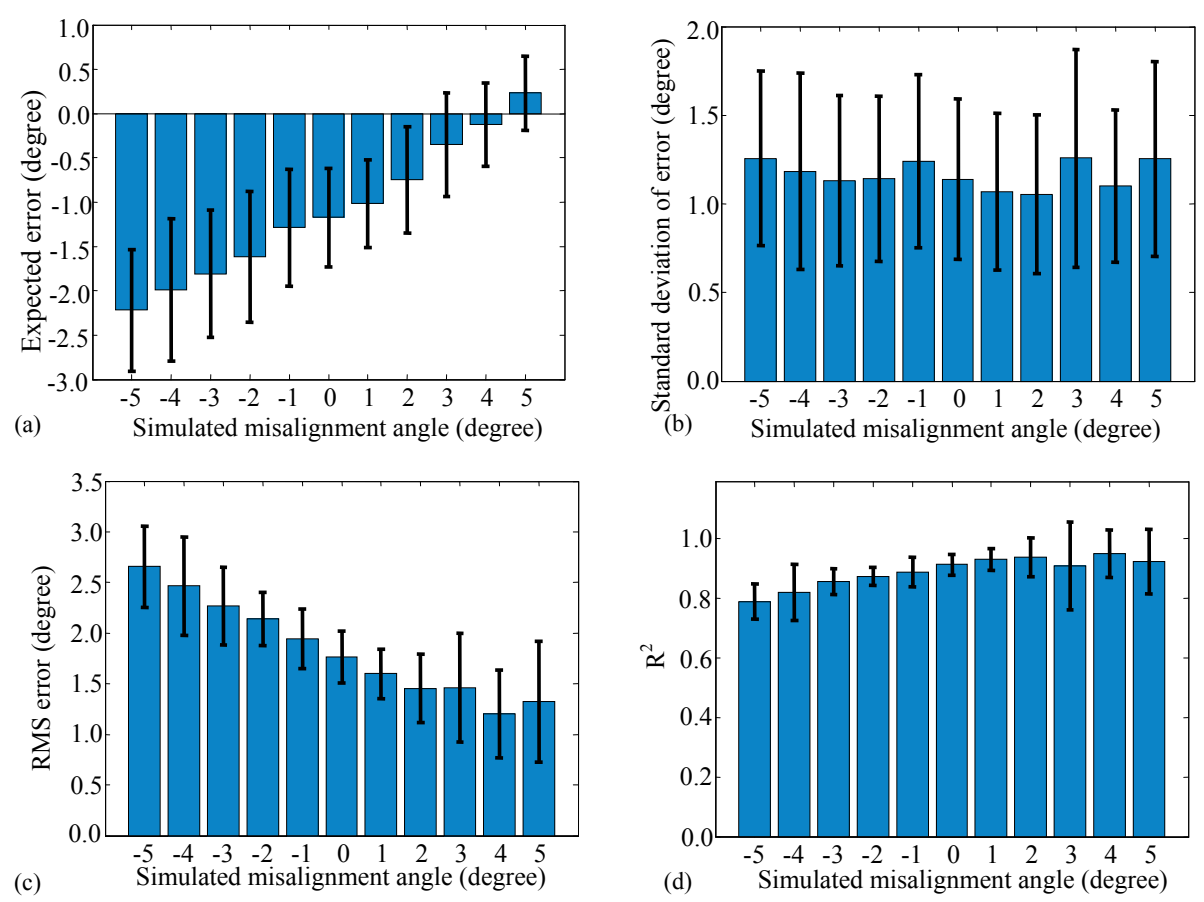

Fig. 7. The effect of magnet-sensor misalignment on the IE angle estimation: (a) mean error, (b) standard deviation of error, (c) RMS error, (d) ${ }^{2}$.

showed proper performance even when using a small percentage of data. In both optimization methods, the calibration could be repeated in the final system if the optimization does not reach an acceptable value, i.e. small function value and Cost in QN and MHACIE respectively.

The magnet-sensor misalignment effect on the referencefree angle estimation was studied by simulating the sensor measurements for a range $-5^{\circ}$ to $+5^{\circ}$ misalignments. Applying the MHACIE optimization, the parameters of the IE estimator were obtained. Comparing to the reference angle, $\mathrm{R}^{2}$ of the obtained estimates in cases of the negative misalignments was decreased by less than 0.13 , suggesting still high correlation between the reference angle and the estimates. The standard deviation of error was not very sensitive to the simulated misalignments, and always remained below $1.3^{\circ}$. Interestingly, the offset error, i.e. expected mean error, even decreased in the cases of positive misalignments. This could be explained by an originally existed few degrees of negative misalignment. Actually, the magnet-sensor alignment performed visually and potentially involved an offset which was partly compensated in the case of positive misalignments. Comparing the estimation error for the simulated magnet-sensor misaligned cases with original estimations errors, the increase in RMS error was $0.9^{\circ}(45 \%)$ in the worst case. In the case of perfect alignment of the magnet and sensor, the frame of sensor, due its axial rotation, might be still misaligned with the orientation of magnetic field at zero IE angle. This would also result in an offset error for the reference-free angle estimators.

Comparing to the reference-dependent estimators, the performances of the obtained reference-free estimators were slightly lower. This is due to the fact that the parameters of the reference-dependent estimators were explicitly derived to optimize the performance of the estimations, i.e. minimize the mean squared error to the reference angles. While the parameters of the reference-free estimators were derived to better maintain the assumed physical relation between the sensor's channels (3). Latter criterion does not imply the optimality of the angle estimator. However, when a smaller portion of data was used for optimization or training (Fig. 6), higher standard deviations were resulted for all performance metrics of the reference-dependent estimators which shows their fragility to the amount of data and the necessity to be trained over rich data. In contrast, the performance indices variations were not changed with the percentage of training (optimization) data for the reference-free estimators (Fig. 5) showed their robustness to the quantity of the data. With more scrutiny on the performances of the reference-free and the reference-dependent estimators, the main difference is in the mean error (offset) which was more than two folds higher in the reference-free case. This offset could be due to the initial misorientation of the AMR sensor according to the magnet axis (Fig. 2). The offset in reference-free IE angle estimates does not affect the estimation of range of angle. The offsets could be adjusted in a post-processing if a single point reference angle measurement is provided, which can improve the estimators' performances significantly. Otherwise, any biomechanical constraint such as the maximum possible angle could be used to reduce the offset error.

Since the magnet and sensor were closely placed in the knee prosthesis no earth magnetic field influence was observed. This was tested prior to the data collection through repeated measurements when the simulator was placed in different orientation on a table. It is also worthy to note that after the integration of the AMR sensor and supplementary electronics into the final PE insert, the insert strength needs to be tested to guarantee the persistence of PE during 20 years. 
The error of the proposed system -offset $<2^{\circ}$ and RMS before and after removing the offset $<2^{\circ}$ and $1.3^{\circ}$ which are smaller than $9.5 \%$ of range of IE- is lower than the previously reported wearable systems. For instance a combination of accelerometers and gyroscopes [13] was obtained an offset error of $3.4 \pm 2.7^{\circ}$ and an RMS error after removing the offset of $1.6 \pm 0.5^{\circ}$, and by adding the magnetic field coils and sensors to the inertial sensors [34], the RMS error of orientation of different segment was reported in range of $\left[2.3^{\circ} 3.2^{\circ}\right]$. It is worthy to note that in both mentioned studies the reference systems were also STA-affected and their reported errors do not thus represent the soft tissue errors. The standard deviation of error of our proposed method is very close to the reported value in the fluoroscopic studies [17].

The proposed calibration method facilitates the use of the instrumented prosthesis for IE angle measurements by relaxing the need for any reference measurement before and after the implantation. The proposed reference-free calibration showed potentials to work in magnet-sensor misaligned cases. The method can be extended for knee 3D angle estimation, by being separately applied to a set of sensors placed in known points, and then finding the orientations of the magnet(s) to be the source for such magnetic field distribution.

\section{REFERENCES}

[1] A. J. Carr, O. Robertsson, S. Graves, A. J. Price, N. K. Arden, A. Judge, and D. J. Beard, "Knee replacement," The Lancet, vol. 379, no. 9823, pp. 1331-1340, Apr. 2012.

[2] D. D. D'Lima, S. Patil, N. Steklov, and C. W. Colwell, "The 2011 ABJS Nicolas Andry Award: 'Lab'-in-a-Knee: In Vivo Knee Forces, Kinematics, and Contact Analysis," Clin. Orthop. Relat. Res., vol. 469, no. 10, pp. 2953-2970, May 2011.

[3] J. J. Collins, "The redundant nature of locomotor optimization laws," J. Biomech., vol. 28, no. 3, pp. 251-267, Mar. 1995.

[4] K. E. Wilk, R. F. Escamilla, G. S. Fleisig, S. W. Barrentine, J. R. Andrews, and M. L. Boyd, "A comparison of tibiofemoral joint forces and electromyographic activity during open and closed kinetic chain exercises," Am. J. Sports Med., vol. 24, no. 4, pp. 518-527, Aug. 1996.

[5] A. Leardini, L. Chiari, U. D. Croce, and A. Cappozzo, "Human movement analysis using stereophotogrammetry: Part 3. Soft tissue artifact assessment and compensation," Gait Posture, vol. 21, no. 2, pp. 212-225, Feb. 2005

[6] D. L. Benoit, D. K. Ramsey, M. Lamontagne, L. Xu, P. Wretenberg, and P. Renström, "Effect of skin movement artifact on knee kinematics during gait and cutting motions measured in vivo," Gait Posture, vol. 24, no. 2, pp. 152-164, Oct. 2006.

[7] A. Barré, J.-P. Thiran, B. M. Jolles, N. Theumann, and K. Aminian, "Soft tissue artifact assessment during treadmill walking in subjects with total knee arthroplasty," IEEE Trans. Biomed. Eng., vol. 60, no. 11, pp. 3131-3140, Jun. 2013.

[8] S. Bhan, R. Malhotra, E. K. Kiran, S. Shukla, and M. Bijjawara, "A Comparison of Fixed-Bearing and Mobile-Bearing Total Knee Arthroplasty at a Minimum Follow-up of 4.5 Years," J. Bone Jt. Surg., vol. 87, no. 10, pp. 2290-2296, Oct. 2005.

[9] B. M. Jolles, A. Grzesiak, A. Eudier, H. Dejnabadi, C. Voracek, C. Pichonnaz, K. Aminian, and E. Martin, "A randomised controlled clinical trial and gait analysis of fixed- and mobile-bearing total knee replacements with a five-year follow-up," J. Bone Joint Surg. Br., vol. 94, no. 5, pp. 648-655, May 2012.

[10] I. P. I. Pappas, T. Keller, S. Mangold, M. R. Popovic, V. Dietz, and M. Morari, "A reliable gyroscope-based gait-phase detection sensor embedded in a shoe insole," IEEE Sens. J., vol. 4, no. 2, pp. 268-274, 2004.

[11] B. Mariani, C. Hoskovec, S. Rochat, C. Büla, J. Penders, and K. Aminian, "3D gait assessment in young and elderly subjects using foot-worn inertial sensors," J. Biomech., vol. 43, no. 15, pp. 29993006, Nov. 2010

[12] J. Barth, J. Klucken, P. Kugler, T. Kammerer, R. Steidl, J. Winkler, J. Hornegger, and B. Eskofier, "Biometric and mobile gait analysis for early diagnosis and therapy monitoring in Parkinson's disease," Int. Conf. IEEE Eng. Med. Biol. Society, EMBC, pp. 868-871, 2011.

[13] J. Favre, B. M. Jolles, R. Aissaoui, and K. Aminian, "Ambulatory measurement of 3D knee joint angle," J. Biomech., vol. 41, no. 5, pp. $1029-1035,2008$.

[14] P. Cheng and B. Oelmann, "Joint-Angle Measurement Using Accelerometers and Gyroscopes: A Survey," IEEE Trans. Instrum. Meas., vol. 59, no. 2, pp. 404-414, 2010.

[15] H. Dejnabadi, B. M. Jolles, and K. Aminian, "A new approach to accurate measurement of uniaxial joint angles based on a combination of accelerometers and gyroscopes," IEEE Trans. Biomed. Eng., vol. 52, no. 8, pp. 1478-1484, 2005.

[16] A. Wixted, M. Portus, W. Spratford, and D. James, "Detection of throwing in cricket using wearable sensors," Sports Technol., vol. 4, no. 3-4, pp. 134-140, 2011.

[17] C. Trozzi, B. L. Kaptein, E. H. Garling, T. Shelyakova, A. Russo, L. Bragonzoni, and S. Martelli, "Precision assessment of model-based RSA for a total knee prosthesis in a biplanar set-up," The Knee, vol. 15 , no. 5 , pp. $396-402$, Oct. 2008.

[18] S. Witvoet-Braam, "Symbios FIRST Knee. Phantom Report. A Tech. Report." Medis Specials, 2007.

[19] B. Heinlein, F. Graichen, A. Bender, A. Rohlmann, and G. Bergmann, "Design, calibration and pre-clinical testing of an instrumented tibial tray," J. Biomech., vol. 40, Supplement 1, pp. S4-S10, 2007.

[20] D. D. D'Lima, S. Patil, N. Steklov, J. E. Slamin, and C. W. Colwell, "Tibial Forces Measured In Vivo After Total Knee Arthroplasty," $J$. Arthroplasty, vol. 21, no. 2, pp. 255-262, Feb. 2006.

[21] A. Arami, M. Simoncini, O. Atasoy, S. Ali, W. Hasenkamp, A. Bertsch, E. Meurville, S. Tanner, P. Renaud, C. Dehollain, P. Farine, B. M. Jolles, K. Aminian, and P. Ryser, "Instrumented Knee Prosthesis for Force and Kinematics Measurements," IEEE Trans. Autom. Sci. Eng., vol. 10, no. 3, pp. 615-624, 2013.

[22] S. Almouahed, M. Gouriou, C. Hamitouche, E. Stindel, and C. Roux, "Design and Evaluation of Instrumented Smart Knee Implant," IEEE Trans. Biomed. Eng., vol. 58, no. 4, pp. 971-982, Apr. 2011.

[23] B. Kirking, J. Krevolin, C. Townsend, C. W. Colwell Jr., and D. D. D'Lima, "A multiaxial force-sensing implantable tibial prosthesis," $J$. Biomech., vol. 39, no. 9, pp. 1744-1751, 2006.

[24] A. Arami, J. Miehlbradt, and K. Aminian, "Accurate internal-external rotation measurement in total knee prostheses: A magnetic solution," J. Biomech., vol. 45, no. 11, pp. 2023-2027, Jul. 2012.

[25] J. Lenz and A. S. Edelstein, "Magnetic sensors and their applications," IEEE Sens. J., vol. 6, no. 3, pp. 631-649, 2006.

[26] C. Hu, M. Li, S. Song, W. Yang, R. Zhang, and M. Q.-H. Meng, “A Cubic 3-Axis Magnetic Sensor Array for Wirelessly Tracking Magnet Position and Orientation," IEEE Sens. J., vol. 10, no. 5, pp. 903-913, 2010.

[27] F. C. Paixão, L. A. Corá, M. F. Américo, R. B. de Oliveira, O. Baffa, and J. R. A. Miranda, "Development of an AMR-ACB Array for Gastrointestinal Motility Studies," IEEE Trans. Biomed. Eng., vol. 59, no. 10 , pp. 2737-2743, 2012 .

[28] E. Raffin, S. Bonnet, and P. Giraux, "Concurrent validation of a magnetometer-based step counter in various walking surfaces," Gait Posture, vol. 35, no. 1, pp. 18-22, Jan. 2012.

[29] A. Barre, J.-P. Thiran, B. M. Jolles, N. Theumann, and K. Aminian, "Semi-automatic segmentation method for roentgen fluoroscopic stereophotogrammetric analysis," presented at the 2nd international RSA meeting, Leiden, Netherlands, 2011.

[30] A. Arami, B. R. Rofoee, and C. Lucas, "Multiple Heterogeneous Ant Colonies with Information Exchange," IEEE Cong. Evolutionary Computation, CEC 2008, pp. 3298-3304, 2008.

[31] J. C. Gilbert, C. Lemarechal, and C. A. Sagastizábal, Numerical Optimization: Theoretical and Practical Aspects. Springer, 2006.

[32] M. Dorigo, V. Maniezzo, and A. Colorni, "Ant system: optimization by a colony of cooperating agents," IEEE Trans. Syst. Man Cybern. Part B Cybern., vol. 26, no. 1, pp. 29-41, 1996.

[33] M. Dorigo and C. Blum, "Ant colony optimization theory: A survey," Theor. Comput. Sci., vol. 344, no. 2-3, pp. 243-278, Nov. 2005.

[34] D. Roetenberg, P. J. Slycke, and P. H. Veltink, "Ambulatory Position and Orientation Tracking Fusing Magnetic and Inertial Sensing," IEEE Trans. Biomed. Eng., vol. 54, no. 5, pp. 883-890, 2007. 